\title{
Localization of Small Metal (Oxide) Particles in SBA-15 Using Bright-Field Electron Tomography
}

\author{
A. H. Janssen, ${ }^{\dagger}$ C.-M. Yang, ${ }^{\ddagger}$ Y. Wang, F. Schiith,, A. J. Koster, ${ }^{\S}$ and K. P. de Jong $* \dagger$ \\ Department of Inorganic Chemistry and Catalysis, Debye Institute, Utrecht University, Sorbonnelaan 16, \\ 3584 CA Utrecht, The Netherlands, Max Planck Institut für Kohlenforschung, Kaiser Wilhelm Platz, 1, \\ D-45470, Mülheim an der Ruhr, Germany, and Department of Molecular Cellbiology, Utrecht University, \\ Padualaan 8, $3584 \mathrm{CH}$ Utrecht, The Netherlands
}

Received: March 24, 2003; In Final Form: May 22, 2003

\begin{abstract}
Small gold $(5 \mathrm{~nm})$ and zirconia $(2-3 \mathrm{~nm})$ particles inside the mesopores of SBA-15 were visualized with bright-field electron tomography. Due to the ordered nature of the mesopores and the related diffraction contrast present in the tilt series, the 3D-reconstruction is difficult to interpret. Unequivocal information has been obtained, however, on the presence of the particles in certain pores, while others pores are empty. This nonuniform distribution of particles over the mesopores explains the two-step desorption isotherm in nitrogen physisorption experiments.
\end{abstract}

\section{Introduction}

In the past decade there has been a huge increase in research on ordered mesoporous materials. ${ }^{1,2}$ With their unique textural properties (uniform pores with diameters of approximately 3-30 $\mathrm{nm}$ ) these materials bridge the gap between (microporous) zeolites on the one side and amorphous meso- and macroporous oxides on the other side. Recently it was shown that a tilt series taken in a bright-field transmission electron microscope gives valuable information on the shape of the mesopores on a mesoscopic scale $(10-1000 \mathrm{~nm}){ }^{3}$ To locate small metal (oxide) particles inside the mesopores, electron tomography, sometimes referred to as three-dimensional transmission electron microscopy (3D-TEM), seems to be the most suitable technique to image these materials in three dimensions. However, one of the basic assumptions in the standard 3D-reconstruction approaches is that image intensity is related to a projection of the mass density (or phase density) of the material. This is a valid assumption for specimen producing scattering- or weak-phase contrast images. Unfortunately, the theory of electron tomography does not account for diffraction contrast in the tilt series. For zeolites the distance between the lattice planes is much smaller than the resolution in the TEM images at low magnification. As a consequence, the diffraction contrast does not hamper the 3D-reconstruction and good results have been obtained on the localization of small silver particles on a zeolite. ${ }^{4}$ For ordered mesoporous materials, the structures that give rise to the diffraction contrast (i.e. the ordered pores) are of the same order of magnitude as the resolution of the 3D-reconstruction. Earlier we showed that TEM tilt series can be very useful for the study of the mesoscopic shape of the mesopores in ordered mesoporous materials. ${ }^{3}$ Here we show the first bright-field electron tomographic reconstruction obtained for ordered mesoporous materials. From the localization of small metal (oxide) particles inside the mesopores we show the possibilities and

\footnotetext{
* Corresponding author. E-mail: k.p.dejong@chem.uu.nl.

† Debye Institute.

$\doteqdot$ Max Planck Institut für Kohlenforschung.

$\S$ Utrecht University.
}

limitations of the use of bright-field electron tomography for ordered mesoporous materials. The obtained results on the location of the particles are compared with nitrogen physisorption experiments.

\section{Experimental Section}

Materials. The synthesis of the SBA-15 sample is described in refs 5-7. A brief description of the synthesis is as follows. The material was prepared by dissolving $4 \mathrm{~g}$ of Pluronic P123 (nonionic triblock copolymer, $\mathrm{EO}_{20} \mathrm{PO}_{70} \mathrm{EO}_{20}$ ) in $150 \mathrm{~mL}$ of a $2 \mathrm{M} \mathrm{HCl}$ solution. Subsequently, TEOS (tetraethyl orthosilicate) was added to obtain a molar ratio of TEOS/surfactant of 59 . The solution was stirred for $8 \mathrm{~h}$ at room temperature and then aged for $16 \mathrm{~h}$ at $353 \mathrm{~K}$. The white solid was filtered, washed, and calcined at $823 \mathrm{~K}$.

The synthesis of $\mathrm{ZrO}_{2}$ inside the mesopores of SBA-15 is described in ref 8 . In short, the $\mathrm{ZrO}_{2}$ particles were synthesized by incipient wetness impregnation of SBA-15 with an acidic aqueous solution of $\mathrm{ZrOCl}_{2}$ and urea. By heating to $90{ }^{\circ} \mathrm{C}$ for $3 \mathrm{~h}$ the urea decomposes under formation of hydroxyl ions, thus causing zirconia to precipitate inside the mesopores. Finally, the samples were calcined.

The synthesis of gold particles inside the mesopores of SBA15 is described in ref 9. Briefly, SBA-15 was first functionalized via silylation (without passivation of the small external surface), thus introducing positively charged groups on the pore surface. Next, gold was introduced by ion exchange from an $\mathrm{HAuCl}_{4}$ solution. After washing and drying, the samples were reduced in hydrogen at $150{ }^{\circ} \mathrm{C}$.

3D-TEM. For electron microscopy, a droplet of a colloidal gold suspension (Sigma, $5 \mathrm{~nm}$ gold) was dried on a coppergrid-supported carbon film, thus providing markers for the alignment of the data set. The sample was suspended in ethanol using ultrasound, after which a droplet of the suspension was dried on this grid. From a representative crystal a tilt series of ca. 141 images was taken from about $+70^{\circ}$ to $-70^{\circ}$ with $1^{\circ}$ intervals at magnifications between $11.5 \mathrm{k}$ and $19 \mathrm{k}$ on a Tecnai 20 microscope $(200 \mathrm{kV})$ with a $2048 \times 2048$ pixel CCD camera 


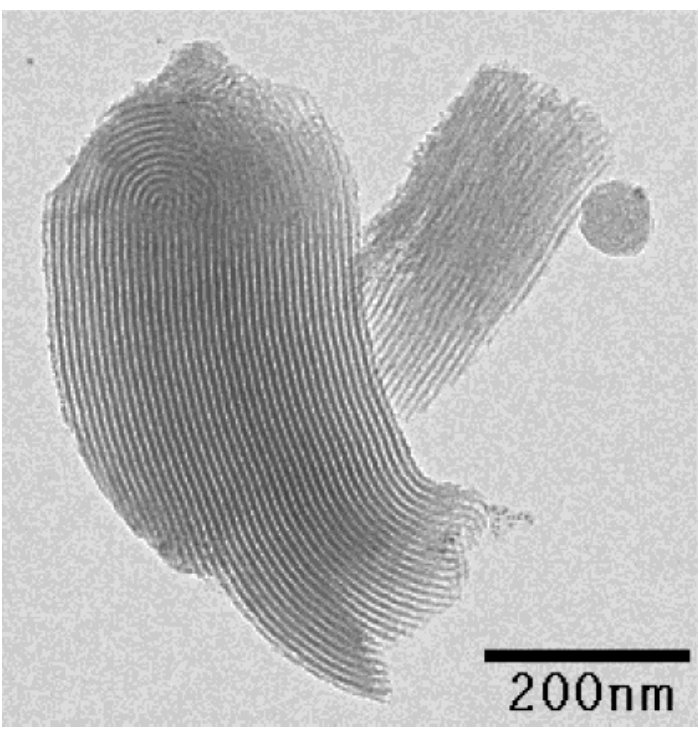

Figure 1. Conventional TEM image of SBA-15.

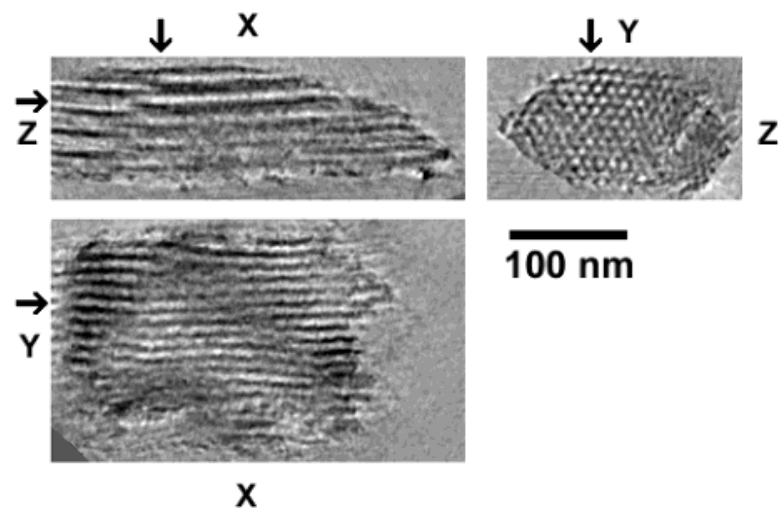

Figure 2. Three orthogonal slices through the 3D-TEM reconstruction of the small SBA-15 particle of Figure 1. Two black arrows next to the $\mathrm{XZ}$ plane indicate the place of the two other crosscuts.

(pixel size $=14 \mu \mathrm{m}$ ), using software for automated electron tomography. To further improve the quality of the reconstruction for an SBA-15 particle without metal (oxide) particles, a second tilt series of the same particle was taken from $+70^{\circ}$ to $-70^{\circ}$ with $1^{\circ}$ increment after the sample grid had been rotated manually over ca. $90^{\circ}$ (double tilt series approach). After alignment of the two tilt series, a combined 3D-reconstruction of the two tilt series was calculated as a stack of thin $(1-2 \mathrm{~nm})$ slices using IMOD. ${ }^{10}$

\section{Results}

In Figure 1 a TEM image of the SBA-15 sample without metal (oxide) particles is shown. The pores in the large particle are U-shaped, whereas the pores in the small particle are straight. In Figure 2 three orthogonal slices through the 3D-TEM (double tilt) reconstruction of the small SBA-15 particle in Figure 1 are given. These slices show that the mesopores are almost straight (XY and XZ) and hexagonally packed (YZ). Figure 3 gives an enlargement of the $X Y$ and $Y Z$ slice from Figure 2 (YZ slice 2) together with another YZ slice (slice 1) through the same 3D-reconstruction. YZ slice 2 shows a hexagonal arrangement of white pores surrounded by black pore walls, whereas YZ slice 1 suggests a hexagonal arrangement of black pores surrounded by white pore walls.

In Figure $4 \mathrm{a}$ a conventional TEM image of the SBA-15 sample with gold particles in the mesopores is shown. Due to

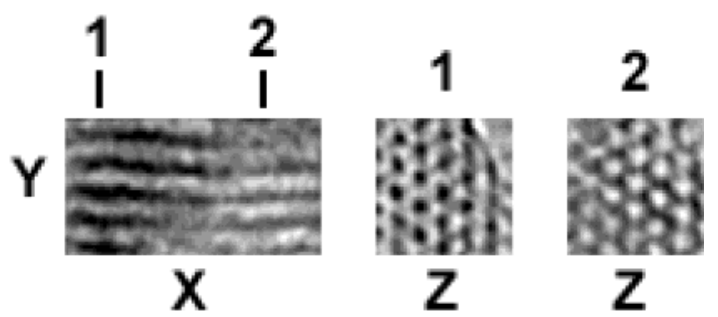

Figure 3. Two $\mathrm{YZ}$ slices $(54 \times 54 \mathrm{~nm})$ through the 3D-TEM reconstruction of Figure 2 showing an apparent change of contrast in the reconstruction.
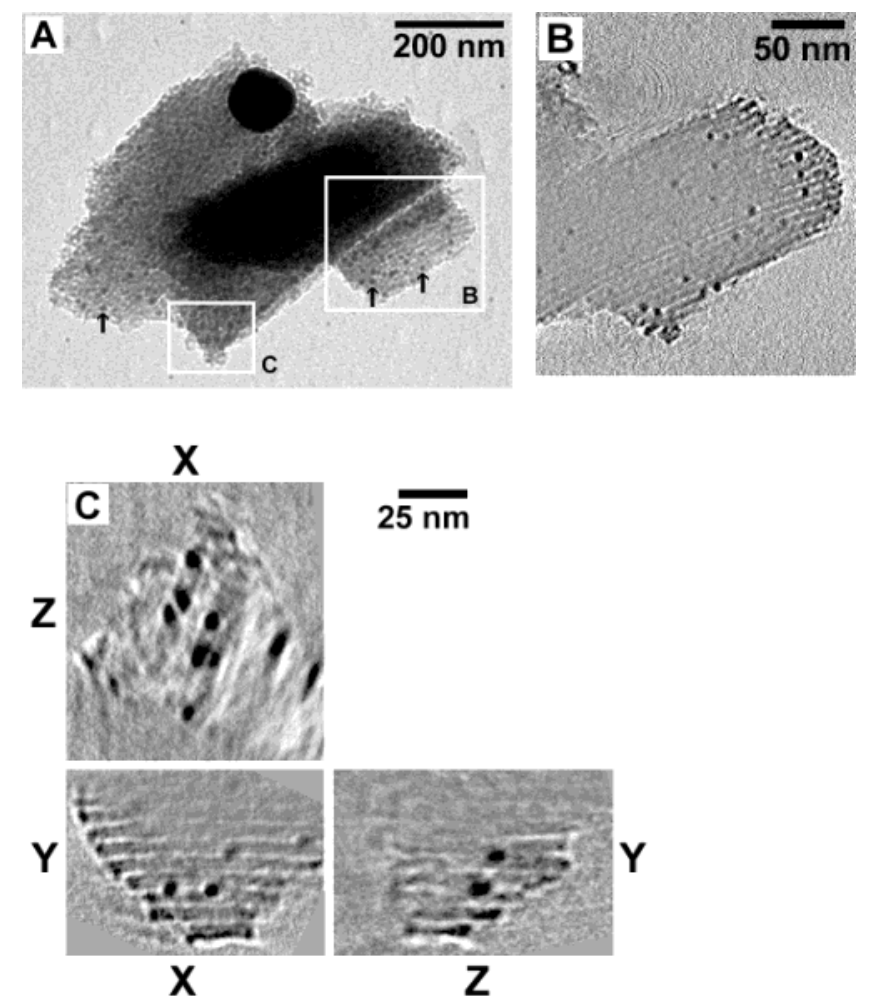

Figure 4. Gold particles in SBA-15: (a) conventional TEM image, (b) slice through the 3D-TEM reconstruction, (c) three orthogonal slices through the 3D-reconstruction.

a different orientation of the pores relative to the electron beam the ordered nature of the SBA-15 material is not as visible as for the specimen in Figure 1. In a movie of a tilt series of this material (see Supporting Information) the ordered nature is evident. Next to one big gold particle, many small gold particles are visible in Figure 4a (some are indicated with an arrow), but their location, inside the mesopores or on the external surface, remains unclear. Next to the SBA-15 particle $5 \mathrm{~nm}$ gold beads are visible that were deposited on the sample grid for the alignment of the tilt series. In Figure $4 \mathrm{~b}$ a slice through the 3D-reconstruction of the right part of the SBA-15 particle of Figure $4 \mathrm{a}$ is shown. The gold particles (black dots) are visible and also the mesopores (white lines) can be distinguished, although they are not clear in the middle of the particle. Figure $4 \mathrm{c}$ shows three orthogonal slices through another part of the 3D-TEM reconstruction. In the XY slice the gold particles inside the mesopores can be distinguished. The gold particles appear to be larger than the white lines, which could suggest that their size exceeds the size of the mesopores. However, one should keep in mind that the diameter of the white line does not necessarily correspond to the diameter of the mesopores (see below). The size of the gold particles is $5-6 \mathrm{~nm}$. In the $\mathrm{XZ}$ 

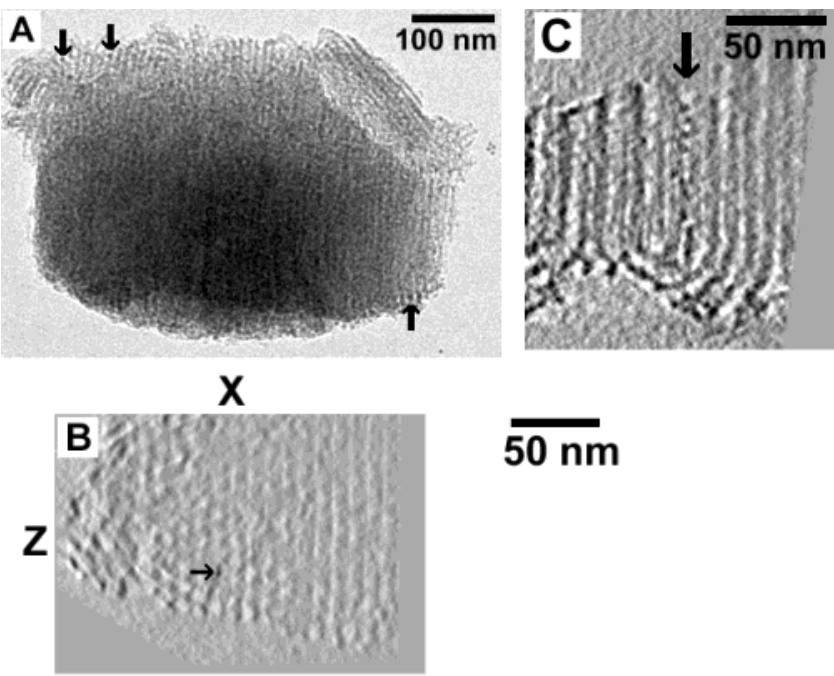

\section{$\overline{50 \mathrm{~nm}}$}

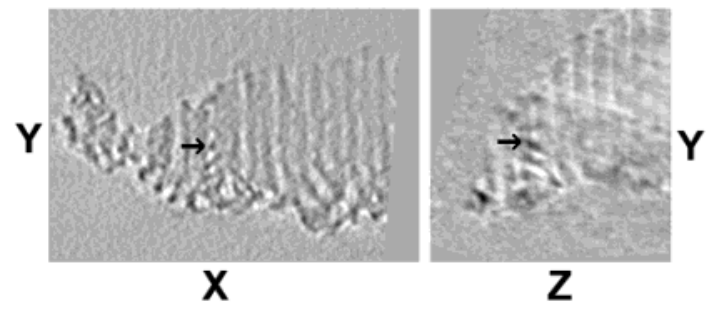

Figure 5. Zirconia particles in SBA-15: (a) conventional TEM image, (b) three orthogonal slices through the 3D-reconstruction (the black arrows indicate the same zirconia particle in the three orientations), (c) slice through the 3D-TEM reconstruction showing U-shaped pores and zirconia particles inside the mesopores (black arrow).

slice the hexagonal packing of the mesopores is not visible and the gold particles are elongated.

The conventional TEM image in Figure 5a shows small $\mathrm{ZrO}_{2}$ particles on SBA-15. The ordered nature of the SBA-15 material is not clear from this image, as the tilt angle of the sample was chosen such that diffraction contrast was (almost) absent, to allow imaging of the small zirconia particles. Some of the largest $\mathrm{ZrO}_{2}$ particles $(5-6 \mathrm{~nm})$ are indicated with an arrow. The ordered nature of the material is evident from a movie of a tilt series of this material (see Supporting Information). In Figure $5 \mathrm{~b}$ three orthogonal slices through a part of the 3D-TEM reconstruction of the SBA-15 particle from Figure 5a are shown. In these three orthogonal slices the zirconia particles are visible as small black dots, whereas the mesopores are visible as lighter regions. The arrows in the three orthogonal slices indicate the same zirconia particle. In Figure 5c another XY slice through the reconstruction is given, showing some pores with many zirconia particles in it, while other pores do not contain zirconia particles. In this $X Y$ slice also the curved nature of the mesopores (U-shape) can be seen.

In Figure 6 the nitrogen adsorption and desorption isotherms of the SBA-15 samples with gold and zirconia particles, respectively, are shown. The isotherm shows a distinct hysteresis loop with a two-step desorption branch. In contrast, the isotherm of the parent material corresponds to the one expected for cylindrical mesopores with a sharp size distribution, i.e., it has a single capillary condensation step. BJH analysis of the desorption branch allows one to calculate a size distribution with the majority of the pores lying between 6.5 and $7.5 \mathrm{~nm}$.

\section{Discussion}

3D-Reconstruction of SBA-15. The three orthogonal slices in Figure 2 through the 3D-reconstruction of the small SBA-15 particle of Figure 1 show that the pores are almost straight and hexagonally ordered. Our eyes interpret the YZ slice in Figure 2 as showing a hexagonal ordering of white pores surrounded by a black pore wall, just as is expected from a system with empty pores. In contrast to this, YZ slice 1 in Figure 3 seems to show a hexagonal ordering of black pores surrounded by white pore walls. However, we postulate that this is not the case but rather a contrast effect due to the diffraction contrast in the original TEM images (below). The white areas in this YZ slice are still the pores and the black areas are still the pore walls. When a single pore is followed from 2 to 1 in the XY slice, the corresponding $\mathrm{YZ}$ slices along that trajectory show the pore always in white. However, going from 2 to 1 in the $\mathrm{XY}$ slice the pore walls become more pronounced in the reconstruction. In $\mathrm{YZ}$ slice 1 these strongly pronounced pore walls are visible as large black spots, rather than a continuous black pore wall around the white pores. This is caused by the assignment of gray areas to belong to either the "white part" or to the "black part" of the image. Our eyes focus strongly at the black spots in YZ slice 1 and easily regard the rest as "white", which leads to a picture of black spots surrounded by white. This is shown in Figure 7a, where a threshold has been applied to the data to show the gray pixels in white. A closer look at YZ slice 1 in Figure 3 reveals that the white spots are surrounded by three black spots that are connected to each other with gray areas. If these gray values are regarded as belonging to the "black part" of the image, one ends up with white spots surrounded by black. This is shown in Figure $7 \mathrm{~b}$, where a threshold (with a different value than for Figure 7a) has been applied to the data. In principle, one would expect the pore walls to have the same gray value (black) everywhere. This is, however, not the case. In YZ slice 2 of Figure 3 the pore walls also differ strongly in gray value, although our eyes interpret the image as white pores surrounded by gray/black pore walls. It is not exactly clear why the pore walls are not uniform in gray value. A likely explanation could be the fact that diffraction contrast plays a role in the image formation of the original TEM images. As has been mentioned in the Introduction, the theory of electron tomography has not been developed for images that have been formed with diffraction contrast. Although it is not exactly clear what the effect of the diffraction contrast is on the final reconstruction, the $X Y$ slice of Figure 3 shows enormous changes in contrast. This is even more clear when the subsequent $X Y$ slices are displayed in a movie (see Supporting Information). The contrast changes seem to run as a wave through the reconstruction.

Crystalline materials give rise to diffraction contrast in certain orientations. Above it was shown that for SBA-15 this can hamper the interpretation of the 3D-reconstruction. However, for zeolite samples the reconstructions show a uniform gray value for the zeolite material. ${ }^{11,12}$ Although zeolites are crystalline and give rise to diffraction contrast in the electron microscope in certain orientations, the distance between the lattice planes (in the $\AA$ range) is smaller than the resolution of the TEM images. Instead of lattice planes being visible in certain orientations, the entire zeolite crystal is imaged somewhat darker in the orientations where diffraction contrast occurs. In the 3DTEM reconstruction this does not result in contrast changes within the zeolite crystal. For ordered mesoporous materials, however, the distance between the lattice planes is in the nanometer range. At the low magnifications used, also the resolution of the TEM images is in the nanometer range. This results in the diffraction-based imaging of the lattice planes in 


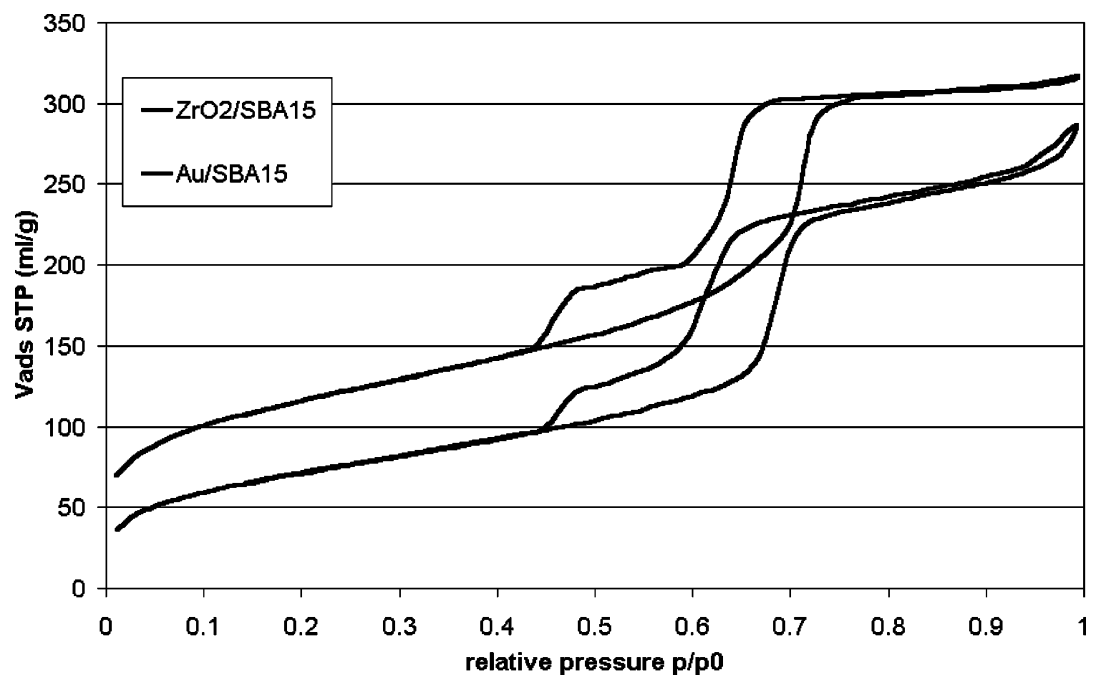

Figure 6. Nitrogen adsorption and desorption isotherms of Au/SBA-15 (bottom) and $\mathrm{ZrO}_{2} / \mathrm{SBA}-15$ (top).

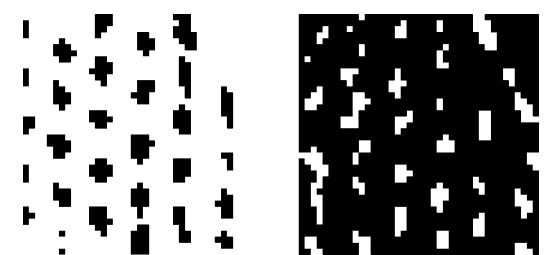

Figure 7. Thresholding of $\mathrm{YZ}$ slice 1 from Figure 3: in part a all gray pixels are converted to white, while in part $b$ all gray pixels are converted to black.

the tilt series, which gives rise to a nonuniform gray value for the pore walls in the calculated 3D-reconstruction.

Location of Metal and Metal Oxide Particles in SBA-15. The slice through the 3D-reconstruction in Figure $4 \mathrm{~b}$ shows the gold particles to be distributed over the sample. However, there is an uneven distribution of the gold particles over the individual pores: some pores contain several gold particles, while other pores are empty. Also the three orthogonal slices through the $3 \mathrm{D}$-reconstruction shown in Figure $4 \mathrm{c}$ show that the gold particles are indeed located in the mesopores of the SBA-15 support. In both the XY and the YZ slice the gold particles (black spots) and the mesopores (white lines) are visible. In both orientations the gold particles are visible inside the SBA15 particle. The middle of the gold particle coincides with the middle of the pore. So, although the hexagonal arrangement of the mesopores is not visible in the $\mathrm{XZ}$ slice, it can still be concluded that the gold particles are present inside the mesopores and not on the external surface of the SBA-15 particle. The hexagonal arrangement of the mesopores in the reconstruction in Figure 3 is much more clear than for this reconstruction. This is caused by the smaller thickness of the sample and the use of a double tilt series for the reconstruction in Figure 3, both of which increase the resolution of the reconstruction.

The fact that in both the XY and YZ slice the diameter of the gold particles is larger than the diameter of the white lines visible in the reconstruction does not necessarily mean that the gold particles have crushed the pore wall locally. The diameter of the white line is much smaller than the diameter expected on the basis of the nitrogen physisorption data. Also the pore wall in the image is much thicker than expected. It was checked that the slice through the 3D-reconstruction cuts through the middle of the pores. Otherwise, if the slice through the 3Dreconstruction cuts through the top of a pore, the width of the white lines will always be smaller than the diameter of the pore and the width of the black lines will, consequently, be larger than the thickness of the pore walls. Apparently, diffraction contrast in the tilt series results in the 3D-reconstruction in an exaggeration of the thickness of the pore walls at the cost of the diameter of the pore. Further evidence for intact mesopores is that the gold particles are rather uniform in size. Their diameter does not exceed $6 \mathrm{~nm}$, which is slightly below the diameter of the mesopores determined with nitrogen physisorption. We do not need to give a reference for the diameter, since we have determined it from the isotherm data. If the pore walls were crushed, also gold particles with diameters larger than the mesopore diameter are expected.

The three orthogonal slices through the reconstruction of the $\mathrm{ZrO}_{2} / \mathrm{SBA}-15$ sample (Figure 5b) show that it is possible to visualize $2-3 \mathrm{~nm} \mathrm{ZrO}_{2}$ particles in the mesopores of SBA-15 in the 3D-reconstruction, despite the occurrence of diffraction contrast in the tilt series. Analogous to the discussion of the $\mathrm{Au} / \mathrm{SBA}-15$ sample above, it can be concluded that the zirconia particles are inside the mesopores, notwithstanding the fact that the particles are elongated in the YZ slice and that the hexagonal arrangement of the mesopores is not visible in the $\mathrm{XZ}$ slice. In the XY slice several zirconia particles are present in the same mesopore, while other mesopores are empty. The size of the zirconia particles is smaller than the pore diameter. Therefore, the pore volume of the mesopore between two zirconia particles in one mesopore is only accessible through a small pore neck. This explains the two-step hysteresis loop in the nitrogen physisorption experiments of these materials (Figure 6). The empty mesopores behave like cylindrical mesopores with a hysteresis at higher relative pressures $\left(\mathrm{p} / \mathrm{p}_{0}=0.6\right)$, whereas the mesopores with zirconia particles inside behave like inkbottle type mesopores with a hysteresis loop that closes around a relative pressure of ca. 0.4. ${ }^{8} \mathrm{~A}$ similar argument holds for the $\mathrm{Au} / \mathrm{SBA}-15$ sample, although here the size of the mesopores is only slightly larger than the size of the gold particles. But even if the mesopores would be fully blocked by the gold particles, the observed double hysteresis loop can still be explained by the fact that SBA-15 contains micropores. If part of the mesopore volume were "blocked" by the gold particles, it would be accessible for nitrogen via the micropores. Similar observations have been reported for modified SBA-15 containing microporous silica plugs that partially block the mesopores. ${ }^{6,7}$

Limits of Bright-Field Electron Tomography. It is clear from Figure $5 \mathrm{~b}$ that it is possible to reveal the three-dimensional location of small metal (oxide) particles in ordered mesoporous materials with bright-field electron tomography. However, the 
$3 \mathrm{D}$-reconstruction is close to the limits of the technique and care has to be taken that artifacts are not misinterpreted. For this type of samples $Z$-contrast tomography seems to be even more powerful. The three-dimensional location of small metal particles inside the mesopores of ordered mesoporous materials have been imaged before using Z-contrast tomography. ${ }^{13,14} \mathrm{With}$ this technique the tilt series is taken with the transmission electron microscope in the scanning mode, while a high angle annular dark field (HAADF) detector is used to collect the images. The use of a HAADF detector has the advantage that the recorded images do not suffer from diffraction contrast. Furthermore, the contrast difference between heavy and light elements is enhanced as the recorded HAADF-STEM signal is approximately proportional to the square of the atomic number $(Z) .{ }^{14,15}$ This allows imaging of very small metal particles in, for example, mesoporous silica. A resolution of $\sim 1 \mathrm{~nm}$ has been obtained for the 3D-reconstruction of small $\mathrm{Pd}-\mathrm{Ru}$ particles in the ordered mesoporous material MCM-41, ${ }^{14}$ which is currently not possible for bright-field electron tomography.

\section{Conclusions}

Bright-field electron tomography of SBA-15-based materials is possible. However, the interpretation of the results can be more difficult, due to diffraction contrast, and care has to be taken that one does not look at artifacts of the reconstruction. New information has been obtained, though, on the location and distribution of metal (oxide) particles in SBA-15 materials. However, for the localization of small metal (oxide) particles on ordered mesoporous materials Z-contrast tomography seems to be even more powerful.

Acknowledgment. Pascal van der Voort and Ellen van Bavel from the University of Antwerp (Belgium) are kindly thanked for the SBA-15 sample.
Supporting Information Available: Movies of the tilt series of SBA-15 (Figure 1), Au/SBA-15 (Figure 4a), and $\mathrm{ZrO}_{2} / \mathrm{SBA}-$ 15 (Figure 5a) and movies of the XY slices of the 3Dreconstructions of an SBA-15 particle (Figure 2) and the $\mathrm{Au} /$ SBA-15 and $\mathrm{ZrO}_{2} / \mathrm{SBA}-15$ samples (Figures $4 \mathrm{~b}$ and $5 \mathrm{~b}$, respectively) are included. This material is available free of charge via the Internet at http://pubs.acs.org.

\section{References and Notes}

(1) Ying, J. Y.; Mehnert, C. P.; Wong, M. S. Angew. Chem., Int. Ed. 1999, 38,56 .

(2) Corma, A. Chem. Rev. 1997, 97, 2373.

(3) Janssen, A. H.; Van Der Voort, P.; Koster, A. J.; De Jong, K. P. Chem. Commun. 2002, 1632.

(4) Koster, A. J.; Ziese, U.; Verkleij, A. J.; Janssen, A. H.; de Jong, K. P. J. Phys. Chem. B 2000, 104, 9368.

(5) Zhao, D.; Feng, J.; Huo, Q.; Melosh, N.; Frederickson, G. H.; Chmelka, B.; Stucky, G. D. Science 1998, 279, 548.

(6) van der Voort, P.; Ravikovitch, P. I.; de Jong, K. P.; Neimark, A. V.; Janssen, A. H.; Benjelloun, M.; van Bavel, E.; Cool, P.; Weckhuysen, B. M.; Vansant, E. F. Chem. Commun. 2002, 1010.

(7) van der Voort, P.; Ravikovitch, P. I.; de Jong, K. P.; Benjelloun, M.; van Bavel, E.; Janssen, A. H.; Neimark, A. V.; Weckhuysen, B. M.; Vansant, E. F. J. Phys. Chem. B 2002, 106, 5873.

(8) Sauer, J.; Kaskel, S.; Janicke, M.; Schüth, F. Stud. Surf. Sci. Catal. 2001, 135, 4740.

(9) Yang, C.-M.; Liu, P.-H.; Ho, Y.-F.; Chiu, C.-Y.; Chao, K.-J. Chem. Mater. 2003, 15, 275

(10) Kremer, J. R.; Mastronarde, D. N.; McIntosh, J. R. J. Struct. Biol. 1996, 116, 71.

(11) Janssen, A. H.; Koster, A. J.; de Jong, K. P. Angew. Chem., Int. Ed. 2001, 40, 1102.

(12) Janssen, A. H.; Koster, A. J.; de Jong, K. P. J. Phys. Chem. B 2002, 106, 11905

(13) Midgley, P. A.; Weyland, M.; Thomas, J. M.; Johnson, B. F. G. Chem. Commun. 2001, 907.

(14) Weyland, M.; Midgley, P. A.; Thomas, J. M. J. Phys. Chem. B 2001, 105, 7882 .

(15) de Jong, K. P.; Koster, A. J. Chem. Phys. Chem. 2002, 3, 776 